\title{
RELACIÓN LONGITUD-PESO, FACTOR DE CONDICIÓN Y TABLA ESTÁNDAR DEL PESO DE MIL ALEVINOS DE GAMITANA Colossoma macropomum (Cuvier, 1818) CRIADOS EN ESTANQUES ARTIFICIALES
}

\author{
Guillermo FELIPA ${ }^{1}$; Walter BLAS ${ }^{1}$; Fernando ALCÁNTARA ${ }^{2}$ \\ 1 Universidad Nacional Federico Villarreal. Facultad de Oceanografía Pesquería, Ciencias Alimentarias y Acuicultura. \\ Escuela Profesional de Ingeniería en Acuicultura. E. mail: guillermo.felipa@gmail.com \\ 2 Instituto de Investigaciones de la Amazonía Peruana. Programa de Investigación para el Uso y Conservación del Agua y \\ sus Recursos. E. mail: fab_0005@hotmail.com
}

\begin{abstract}
RESUMEN
La gamitana (Colossoma macropomum) es un pez amazónico ampliamente cultivado en Sudamérica, debido a su rusticidad, rápido crecimiento en cautiverio, su régimen omnívoro y la alta demanda de su carne en el mercado regional. Conocer la relación entre la longitud y el peso de los alevinos ayudaría a los proveedores de semilla a reducir el manipuleo y estrés de los mismos en el momento de atender a los clientes en los Centros de Producción de Alevinos. El objetivo del estudio fue determinar la relación longitud-peso, el factor de condición y elaborar una tabla estándar del peso de mil alevinos de gamitana criados en estanques artificiales. Se sembraron 3000 post larvas de 10 días de edad en tres estanques de $10 \mathrm{~m}$ x $5 \mathrm{~m}$ x $1 \mathrm{~m}$ fertilizados con gallinaza, cal hidratada y abono verde. Se ofreció alimento artificial con un tenor de proteína de $45 \%$ por un período de tres semanas y luego fue reducido este nivel a $32 \%$ hasta la finalización del experimento. Se efectuaron evaluaciones de crecimiento con una frecuencia de 6 días por un periodo total de 54 días. La longitud de los alevinos fue determinada mediante una cámara digital y el uso del software ImageJ y el peso fue determinado mediante una balanza digital con precisión a $0.02 \mathrm{~g}$. En cada ocasión fueron evaluados 60 alevinos para determinar los promedios que fueron usados para la comparación de medias y determinación de la relación longitud-peso y factor de condición. Fue determinada la ecuación $Y=36.951 \mathrm{x}^{2.4725}\left(\mathrm{R}^{2}=0.9901\right)$ con la cual fue calculada la tabla estándar del peso de mil alevinos. Los alevinos presentaron un crecimiento de tipo alométrico y el factor de condición indicó una declinación paulatina de la condición de la especie que puede deberse a la escasez de alimento y a los altos niveles de densidad de carga.
\end{abstract}

PALABRAS CLAVE: Relación longitud-peso alevinos Colossoma macropomum.

\section{LENGTH-WEIGHT, RELATIONSHIP CONDITION FACTOR AND STANDARD TABLE OF THE WEIGHT FOR ONE THOUSAND BLACK-FINNED PACU FINGERLINGS Colossoma macropomum (Cuvier, 1818) RAISED IN ARTIFICIAL PONDS}

\begin{abstract}
Black-finned pacu (Colossoma macropomum) is an Amazonian fish vastly cultured in South America due to its rusticity, fast growth, omnivore regime and high regional demand for its meat. Assessing the weightlength relationship could be helpful to fingerlings suppliers in reducing hand manipulation and stress of fish during the time of delivery to clients at the hatcheries. This study was performed in order to determine the length-weight ratio, condition factor and develop a standard weight table of a thousand gamitana fingerlings Colossoma macropomum raised in artificial ponds. They were stocked 3000 larvae of 10 days of age in three ponds of $10 \times 5 \times 1 \mathrm{~m}$ fertilized with manure, hydrated lime and green manure. It was featured artificial food with a protein content of $45 \%$ for a period of three weeks and then this level was reduced to $32 \%$ until the end of the experiment. They were growth assessments made with a frequency of 6 days for a total period of 54 days long. The length of the fry was determined by a digital camera and using the software ImageJ 1.46 version and weight was determined by a digital balance accurate to $0.02 \mathrm{~g}$. Each time 60 fingerlings were evaluated to determine the averages that were used to compare means and determining the ratio length-weight and its condition factor. It was determined the equation $\mathrm{Y}=36.951 \mathrm{x}^{2.4725}\left(\mathrm{R}^{2}=0.9901\right)$ with which it was calculated the standard table of the weight of a thousand fingerlings. The fingerlings showed allometric growth and condition factor indicated a gradual decline in the status of species that may be due to food shortages and high charge density.
\end{abstract}

KEYWORDS: Length-weight relationship, fingerlings, Colossoma macropomum. 


\section{INTRODUCCIÓN}

La gamitana (Colossoma macropomum) es una de las especies de escama de gran demanda en la región amazónica del país. En el medio natural puede alcanzar más de un metro de longitud y $30 \mathrm{~kg}$ de peso (Araujo-Lima \& Goulding 1997). Tiene un régimen alimenticio omnívoro, con alto consumo de frutos, hojas y flores en época de expansión del ambiente acuático, que alterna con zooplancton en la época de retracción (Honda, 1974; Araujo-Lima \& Goulding 1997). Esta especie presenta dientes molariformes que le permiten triturar frutos, semillas y caracoles diversos (Kubitza, 2004). En condiciones de cultivo, acepta alimentos artificiales con rendimientos de 6 a 8 toneladas por hectárea por año (Lovshin, 1980; Alcántara et al., 2000a, 2000b y 2000c; Alcántara \& Colace 2001; Arbeláez-Rojas et al., 2002; Alcántara et al., 2006).

El Instituto de Investigaciones de la Amazonía Peruana, viene produciendo, desde hace más de dos décadas, post larvas y alevinos en condiciones controladas para su distribución con fines de cría en estanques de productores. Sin embargo, los alevinos son criados en altas densidades de carga y son transferidos progresivamente a los productores sin conocimiento de su longitud, peso y condición, realizando el conteo de forma individual, con el riesgo que esto tiene para los alevinos y su sobrevivencia.

En el contexto anotado, el estudio tuvo como objetivo analizar la relación longitud-peso y el factor de condición de los alevinos de gamitana criados en estanques artificiales para la propuesta de una tabla estándar del peso de mil alevinos de diversas longitudes.

\section{MATERIALES Y MÉTODOS}

El estudio fue realizado en el Centro de Investigaciones de Quistococha del Instituto de Investigaciones de la Amazonía Peruana (IIAP), situado en el kilómetro 4.5 de la Carretera IquitosNauta, Centro Poblado de Quistococha, Distrito de San Juan, Provincia de Maynas, Región Loreto, en el período comprendido entre el 5 de febrero y el 13 de marzo de 2012.

Fueron utilizados tres estanques de $10 \mathrm{~m}$ x $5 \mathrm{~m} \mathrm{x}$ $1 \mathrm{~m}$ fertilizados con $1000 \mathrm{~kg} \cdot \mathrm{ha}^{-1}$ de gallinaza, 1000 kg.ha ${ }^{-1}$ de cal hidratada y $1500 \mathrm{~kg} \cdot \mathrm{ha}^{-1}$ de abono verde, constituido por hierbas de corte del entorno de los estanques, siguiendo el protocolo establecido con fines de larvicultura en el IIAP (Alcántara, 2010). Los estanques fueron sembrados con 3000 post larvas de C. macropomum de 10 días de edad, cada uno, producidas en laboratorio mediante reproducción inducida $\mathrm{y}$, por tanto, fueron genéticamente semejantes.

La fertilización de los estanques con gallinaza, cal hidratada y abono verde propició el desarrollo del plancton constituyendo el alimento inicial de las post larvas. Adicionalmente, desde la primera semana, se ofreció alimento artificial finamente tamizado, de tal manera que el tamaño de partícula fuera menor de 500 micrones.

El alimento ofrecido en esta etapa estuvo compuesto por harina de pescado $(50 \%)$, harina de soya $(25 \%)$, polvillo de arroz $(24.9 \%)$ y suplemento vitamínico Minevit $(0.1 \%)$, con un tenor de proteína de $45 \%$ por un periodo de tres semanas. Al finalizar la tercera semana, se ofreció un alimento compuesto por harina de pescado $(25 \%)$, harina de soya $(25 \%)$, polvillo de arroz (25\%), harina de maíz (24.9\%) y Minevit $(0.1 \%)$ con un tenor de proteína de 32\% según Alcántara et al. (2010).

Los alevinos fueron evaluados a partir del día doce, con una frecuencia de seis días y en cada ocasión fue considerada la determinación de la longitud y el peso de 60 alevinos, siendo ejecutadas ocho evaluaciones en total. La longitud fue determinada mediante una cámara digital Panasonic DMC-ZS10, utilizando el software ImageJ versión 1.46, que es una aplicación del software de Java para procesamiento y análisis de imágenes, desarrollado por Wayne Rasband en los Institutos Nacionales de Salud de los Estados Unidos (Bourne, 2010). Se midió y pesó cada alevino, usando una balanza digital Camry (EHA701) de 200 g con precisión de $0.02 \mathrm{~g}$.

Se determinó la relación longitud peso, usando la relación exponencial $\mathrm{y}=\mathrm{ax}^{\mathrm{b}}$, de acuerdo a Vazzoler (1982), donde "y" es la variable dependiente, "a" es el intercepto con el eje de la ordenada, "x" la variable independiente y " $\mathrm{b}$ " el exponente de relación entre las variables.

De las ocho evaluaciones del peso ejecutadas de mil alevinos de diversas longitudes, se determinó la relación exponencial entre las variables longitud y peso, de acuerdo a Vazzoler (1982). Con los coeficientes de la ecuación se calculó la tabla estándar del peso de mil alevinos para las longitudes comprendidas entre 2.5 y $5.6 \mathrm{~cm}$.

La ecuación de relación entre la longitud y el peso de mil alevinos fue validada determinando el peso de 1000 post larvas y alevinos para las longitudes comprendidas entre 2.5 y $5.6 \mathrm{~cm}$, contrastando la cifra con el recuento de los alevinos estimados mediante la ecuación, tomando muestras en cada una de las unidades de cultivo. Con la diferencia fue calculado el margen de error y expresado en porcentaje. 
Se determinó también el factor de condición de los alevinos, utilizando los promedios de las observaciones de longitud y peso del periodo de estudio en base a la ecuación $\mathrm{K}=\mathrm{W} \cdot \mathrm{L}^{-3}$, donde $\mathrm{K}$ es el factor de condición, W es el peso y L la longitud del alevino (Vazzoler, 1982). El factor de condición fue multiplicado por 100 para mejorar su visualización.

Se evaluaron también las variables oxígeno disuelto, temperatura, $\mathrm{pH}$ y nitrógeno amoniacal como indicadores de calidad de agua de los estanques de larvicultura y alevinaje. El oxígeno disuelto fue evaluado mediante un Oxímetro YSI 55 Dissolved Oxigen con sensibilidad de $0.1 \mathrm{mg} . \mathrm{L}^{-1}$ y rango de 0 a $10 \mathrm{mg} . \mathrm{L}^{-1}$. La temperatura y el $\mathrm{pH}$ fueron determinados mediante el Potenciómetro WTW, con sensibilidad de 0.004 y rango de 0 a $60{ }^{\circ} \mathrm{C}$ y 0 a 14 respectivamente y el nitrógeno amoniacal fue analizado mediante un kit de Lamotte.

La información sobre la longitud y el peso fue registrada en hojas de cálculo de Excel y los datos fueron procesados usando el programa estadístico SPSS versión 18 , determinando la media, valores mínimos y máximos, desviación típica, coeficiente de correlación y comparación de medias.

\section{RESULTADOS Y DISCUSIÓN}

Los análisis de comparación de medias y análisis de varianza de los promedios de crecimiento en longitud y en peso de las unidades de cultivo $(\mathrm{N}=3)$, no evidenciaron diferencia significativa $(\mathrm{p}>0.05)$, lo que indica un crecimiento similar en los ambientes de cría (Tablas 1 y 2).

La relación entre la longitud $(\mathrm{cm})$ y el peso $(\mathrm{g}) \mathrm{de}$ un millar de alevinos de gamitana C. macropomum criados en estanques artificiales es descrita por la ecuación $\mathrm{Y}=36.951 \mathrm{X}^{2.4725}\left(\mathrm{R}^{2}=0.9901\right)$. Figura 1 . En el estudio fue observado que el valor del exponente fue diferente a 3 lo que indica un crecimiento de tipo alométrico (Ricker, 1975). Nehemia et al. (2012) indican que el factor de condición por debajo de 3 es de tipo alométrico negativo y sobre este valor es alométrico positivo. Por nuestra parte señalamos que el factor de condición de alevinos de C. macropomum, en las condiciones experimentales, es alométrico positivo con una tendencia negativa a través del periodo experimental. El valor del exponente $\mathrm{b}$ determinado en nuestro trabajo es cercano a los valores encontrados por Sousa et al. (2016) en sus grupos experimentales (2.74 y 2.59), en los que utilizó dietas artificiales con un tenor proteico de 28 y $32 \%$, $\left(\mathrm{R}^{2}=0.960\right.$ y 0.958 respectivamente $)$. Adicionalmente, coincidimos con Sousa et al. (2016) en la calificación del crecimiento de tipo alométrico de la especie en estudio. Al respecto, Correia \& Freitas (2013), analizando la relación longitud peso de Colossoma macropomum desembarcado en Manacapurú, Amazonas, luego de determinar que el valor del exponente $b$ es de 2.72 concluyeron que la especie tiene un crecimiento de tipo alométrico negativo, que coincide con nuestro trabajo.

La ecuación que relaciona la longitud y el peso de un millar de alevinos de diversas longitudes de $C$. macropomum fue validada haciendo determinaciones del peso de los alevinos y contando su número individualmente. El análisis estadístico de los resultados evidenció un margen de error comprendido entre 1.1 y $4.8 \%$ por encima del valor calculado con un coeficiente de variación de $1.5 \%$, considerado aceptable que valida la ecuación determinada (Tabla 3 ).

Tabla 1. Comparación de medias de longitud $(\mathrm{cm})$ de alevinos de Colossoma macropomum criados en estanques artificiales.

\begin{tabular}{cccccccc}
\hline Unidad & Media & N & Desv. típ. & Mínimo & Máximo & Varianza & $\begin{array}{c}\text { Error típ. } \\
\text { de la media }\end{array}$ \\
\hline I & 3,9011 & 8 & 1,43013 & 1,68 & 5,69 & 2,045 & 0,50563 \\
II & 3,8638 & 8 & 1,42451 & 1,79 & 5,70 & 2,029 & 0,50364 \\
III & 3,9371 & 8 & 1,37890 & 1,96 & 5,68 & 1,901 & 0,48751 \\
\hline Total & 3,9007 & 24 & 1,34895 & 1,68 & 5,70 & 1,820 & 0,27535 \\
\hline
\end{tabular}

$p>0,05$ 
Tabla 2. Comparación de medias de peso $(\mathrm{g})$ de alevinos de Colossoma macropomum criados en estanques artificiales.

\begin{tabular}{cccccccc}
\hline Unidad & Media & N & Desv. típ. & Mínimo & Máximo & Varianza & $\begin{array}{c}\text { Error típ. } \\
\text { de la media }\end{array}$ \\
\hline I & 1,3180 & 8 & 0,88201 & 0,13 & 2,42 & 0,778 & 0,31184 \\
II & 1,2648 & 8 & 0,87937 & 0,15 & 2,41 & 0,773 & 0,31090 \\
III & 1,3156 & 8 & 0,87016 & 0,18 & 2,40 & 0,757 & 0,30765 \\
\hline Total & 1,2995 & 24 & 0,83856 & 0,13 & 2,42 & 0,703 & 0,17117 \\
\hline
\end{tabular}

$p>0,05$

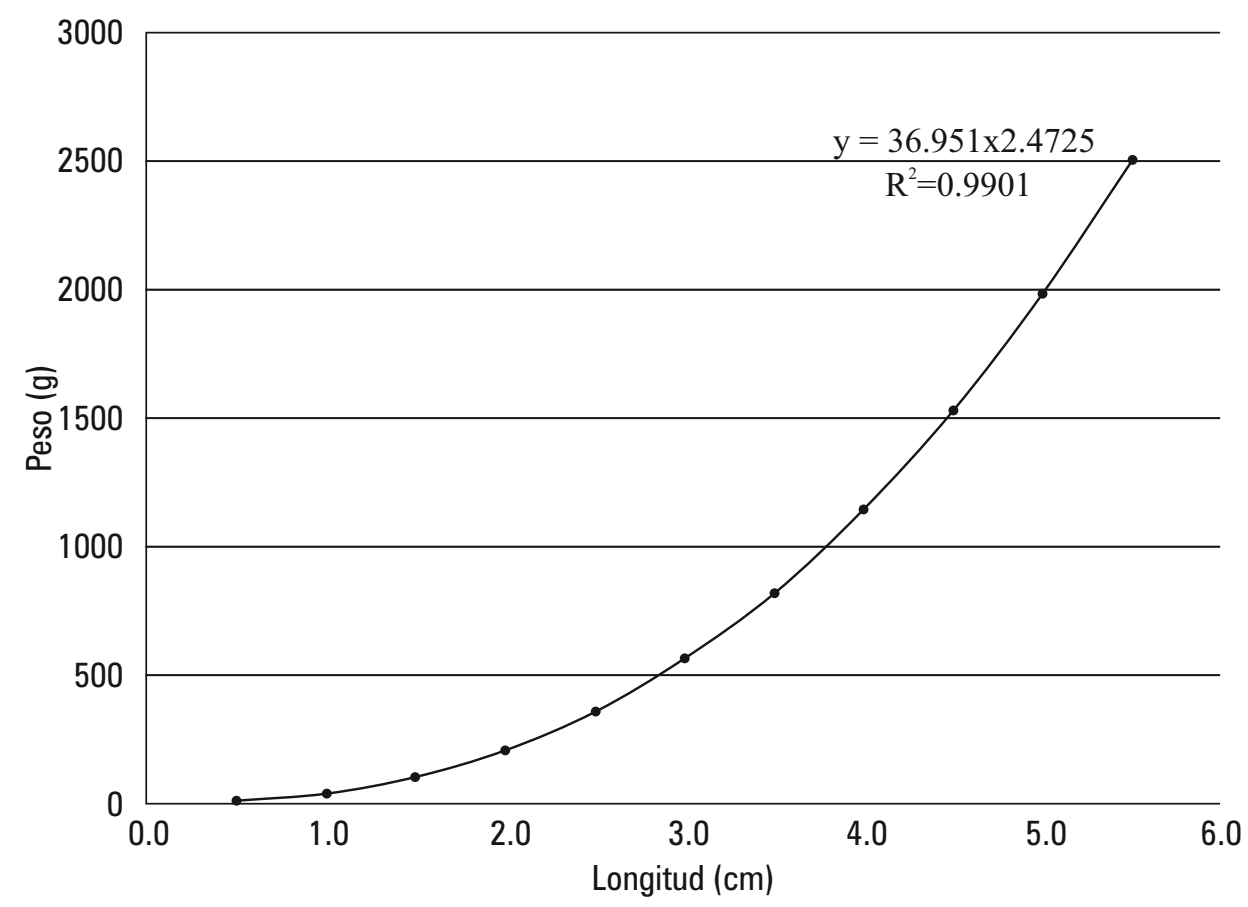

Figura 1. Relación entre la longitud y el peso promedio de 1000 alevinos de gamitana Colossoma macropomum.

Tabla 3. Validación de la estimación del número mediante la determinación del peso de un millar de alevinos de Colossoma macropomum criados en estanques

\begin{tabular}{cccccc}
\hline Estanque & $\begin{array}{c}\text { Longitud } \\
\text { prom. (cm) }\end{array}$ & $\begin{array}{c}\text { Peso de 1000 } \\
\text { alevinos } \mathbf{( g )}\end{array}$ & $\begin{array}{c}\text { Número de } \\
\text { alevinos contados }\end{array}$ & $\begin{array}{c}\text { Diferencia } \\
\text { de 1000 }\end{array}$ & Error \\
\hline I & 5,3 & 2309 & 1048 & 48 & 4,8 \\
II & 5,4 & 2418 & 1011 & 11 & 1,1 \\
III & 5,3 & 2309 & 1032 & 32 & 3,2 \\
\hline
\end{tabular}


En el estudio del factor de condición, fueron observados valores cercanos a 3 al inicio del período de estudio, con una declinación paulatina que indicaría escasez de alimento ligado a la alta densidad de carga $\left(60\right.$ peces. $\left.\mathrm{m}^{-2}\right)$ y al cultivo sin adelgazamiento de la población en la etapa de crecimiento de los alevinos (Tabla 4).

No hemos registrado antecedentes de estudios de relación longitud peso de peces al estado de alevino. Si bien es cierto que en el estudio de la relación longitud-peso de una especie, las variables son presentadas en forma exponencial, indicando a la vez el coeficiente de correlación o de determinación que indica la proximidad o dispersión de las observaciones de campo con la curva de ajustamiento, estos estudios están basados mayormente en la medición de individuos juveniles o adultos y no es frecuente en alevinos. Son frecuentes; sin embargo, los estudios que consideran especímenes juveniles y adultos en los que fue estudiada la relación longitud estándar y longitud total con el peso con fines de administración pesquera (Muto et al., 2000; Morris, 2005; Stewart et al., 2009; Nehemia et al., 2012; Duttra et al., 2012, entre otros). En este sentido, es difícil establecer comparaciones de nuestros resultados con otros trabajos aun cuando reconocemos que la relación longitud peso de una especie es única sea que se trate del estado de alevino, juvenil o adulto.

La ecuación determinada permitió calcular los pesos para las longitudes teóricas comprendidas entre 2.5 y $5.6 \mathrm{~cm}$ y puede ser utilizada para la estimación del número de alevinos en las operaciones de transferencia, haciendo los ajustes correspondientes debido a que el peso es una variable que puede ser influenciada por factores externos como son la densidad de carga, la calidad y cantidad de alimento ofrecido, la frecuencia de alimentación a través del periodo de cría, el tipo y tamaño de partícula, la calidad de agua, entre otros, lo que se refleja en la variación del factor de condición a través del periodo experimental.

La tendencia de disminución del factor de condición a través del periodo experimental observada, sustenta la necesidad de vigilar la condición de los alevinos a través del periodo de cría para mantener su calidad en óptimas condiciones, por sus repercusiones en el proceso de producción y sobrevivencia general posterior en los estanques de producción. Si bien, estos alevinos fueron mantenidos con la densidad de carga inalterable, la observación efectuada sobre la pérdida de condición a través del periodo de crecimiento debe tenerse en cuenta como una señal de advertencia y puede ser contrarrestada con la ejecución de ajustes periódicos de la oferta de alimento, el cuidado de la calidad de agua y la práctica de adelgazamiento progresivo de la población de los estanques de alevinaje con la finalidad de asegurar su calidad en las operaciones de trasferencia para su cultivo a nivel comercial.

El periodo de alevinaje de C. macropomum dura hasta los $10 \mathrm{~cm}$ y es fácilmente reconocible por la presencia del ocelo de color negro (Figura 2). en la parte media de los planos laterales del cuerpo (Alcántara \& Guerra 1986). Sobre los $10 \mathrm{~cm}$ los alevinos pierden el ocelo adquiriendo las características de juveniles.

El uso de la tabla estándar del peso de un millar de alevinos permitirá a futuro la estimación de su número, reduciendo el manipuleo, la mano de obra y sus implicancias en la sobrevivencia general en el proceso de cultivo en los estanques de los productores (Tabla 5).

La temperatura de los ambientes de cultivo de los alevinos fue de $28 \pm 0.5^{\circ} \mathrm{C}$, el pH fue de $6.5 \pm 0.3$; el oxígeno disuelto fue de $5.34 \pm 0.61 \mathrm{mg} . \mathrm{L}^{-1}$ y el nitrógeno amoniacal fue menor que $1 \mathrm{mg} . \mathrm{L}^{-1}$; la dureza fue de $20 \mathrm{mg} . \mathrm{L}^{-1}$ y la alcalinidad fue de 30 mg. $\mathrm{L}^{-1}$ niveles adecuados para el desarrollo de las post larvas y alevinos.

Finalmente, recomendamos efectuar estudios similares para las especies que tienen demanda actual en el mercado amazónico como son, paco Piaractus brachypomus, sábalo cola roja Brycon erythropterum y boquichico Prochilodus nigricans.

Expresamos nuestro agradecimiento al Instituto de Investigaciones de la Amazonía Peruana y en particular al Ing. M. Sc. Salvador Tello Director del Programa de Investigación para el Uso y Conservación del Agua y sus Recursos del IIAP por el financiamiento de esta investigación y por habernos permitido la ejecución del trabajo en las instalaciones del programa en el Centro de Investigaciones de Quistococha. Asimismo, expresamos nuestro agradecimiento a los profesionales y técnicos del programa en mención por su apoyo en el manejo del material biológico. 
Tabla 4. Factor de condición de alevinos de gamitana Colossoma macropomum criados en estanques artificiales.

\begin{tabular}{ccccc}
\hline Muestreo & Long. (cm) & Peso (g) & K & K x 100 \\
\hline 1 & 1,68 & 0,133 & 0,03 & 2,80 \\
2 & 2,43 & 0,356 & 0,02 & 2,46 \\
3 & 3,14 & 0,633 & 0,02 & 2,03 \\
4 & 3,68 & 1,112 & 0,02 & 2,23 \\
5 & 4,32 & 1,722 & 0,02 & 2,13 \\
6 & 4,76 & 1,921 & 0,02 & 1,77 \\
7 & 5,48 & 2,247 & 0,01 & 1,37 \\
8 & 5,69 & 2,420 & 0,01 & 1,31 \\
\hline
\end{tabular}

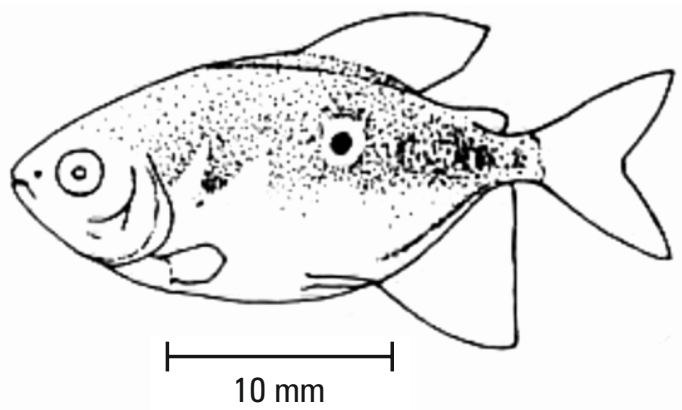

Figura 1. Alevino de gamitana Colossoma macropomum.

Tabla 5. Tabla estándar del peso de 1000 alevinos de diversas longitudes.

\begin{tabular}{cc}
\hline $\begin{array}{c}\text { Longitud } \\
\text { promedio } \\
(\mathbf{c m})\end{array}$ & $\begin{array}{c}\text { Peso de } \\
\mathbf{1 0 0 0} \text { alevinos } \\
\text { (g) }\end{array}$ \\
\hline 2.5 & 356 \\
\hline 2.6 & 392 \\
2.7 & 431 \\
2.8 & 471 \\
2.9 & 514 \\
3.0 & 559 \\
3.1 & 606 \\
3.2 & 656 \\
3.3 & 707 \\
\hline 3.4 & 762 \\
3.5 & 818 \\
\hline 3.6 & 877 \\
3.7 & 939 \\
\hline 3.8 & 1003 \\
\hline 3.9 & 1069 \\
\hline 4.0 & 1138 \\
\hline
\end{tabular}

\begin{tabular}{cc}
\hline $\begin{array}{c}\text { Longitud } \\
\text { promedio } \\
(\mathbf{c m})\end{array}$ & $\begin{array}{c}\text { Peso de } \\
\mathbf{1 0 0 0} \text { alevinos } \\
\text { (g) }\end{array}$ \\
\hline 4.1 & 1210 \\
\hline 4.2 & 1284 \\
4.3 & 1361 \\
\hline 4.4 & 1441 \\
\hline 4.5 & 1523 \\
\hline 4.6 & 1608 \\
\hline 4.7 & 1695 \\
\hline 4.8 & 1786 \\
\hline 4.9 & 1879 \\
\hline 5.0 & 1976 \\
\hline 5.1 & 2075 \\
\hline 5.2 & 2177 \\
\hline 5.3 & 2282 \\
\hline 5.4 & 2390 \\
\hline 5.5 & 2501 \\
\hline 5.6 & 2615 \\
\hline
\end{tabular}




\section{BIBLIOGRAFÍA CITADA}

Alcántara, F.; Guerra, F. 1986. Aspectos de alevinaje de las principales especies nativas utilizadas en piscicultura en la Amazonía peruana. Folia Amazónica, 1(2): 139-161.

Alcántara, F., Kohler, C., Kohler, S., Camargo, W. 2000. Cultivo de gamitana y paco en diferentes densidades. Informe interno. Instituto de Investigaciones de la Amazonía Peruana. PD/A CRSP. Southern Illinois University at Carbondale. Fisheries \& Illinois Aquaculture Center. Iquitos. Perú. 24pp.

Alcántara, F.; Kohler, C.; Kohler, S.; Camargo, W. 2000. Cartilla de Acuacultura en la Amazonía. Instituto de Investigaciones de la Amazonía Peruana. PD/A CRSP. Southern Illinois University at Carbondale. Fisheries \& Illinois Aquaculture Center. Iquitos. Perú. 47pp.

Alcántara, F.; Padilla, P.; Ismiño, R.; Kohler, C.; Kohler, S.; Camargo, W.; De Jesús, M. 2000. Comparative pond culture production of Piaractus brachypomus and Colossoma macropomum. Pond Dynamics/Aquaculture CRSP, Oregon State University. Corvallis OR. US. 10pp.

Alcántara, F.; Colace, B. M. 2001. Piscicultura, seguridad alimentaria y desarrollo sostenible en la carretera Iquitos Nauta y el Río Tigre. Valorando y preservando nuestros peces amazónicos. Programa de seguridad alimentaria para unidades productivas familiares de la carretera Iquitos Nauta y el río Tigre. Unión Europea. Terra Nuova. Instituto de Investigaciones de la Amazonía Peruana. Iquitos. Perú. 83pp.

Alcántara, F.; Chu-Koo, F.; Tello, S.; Kohler, C.; Camargo, W. 2006. Cultivo de peces nativos en la Amazonía peruana. (http://pacrc.uhh.haeaii. edu/mexico/files/bmp/tobey haws/culture native fishes.pdf). Acceso: 10/12/2015.

Alcántara, F.; Rodríguez, L.; Chu-Koo, F.; Napuchi, J.; Tello, S. 2010. Protocolo de cría de alevinos de gamitana, paco y boquichico. Proyecto Cátedra CONCyTEC en Acuicultura. Reproducción inducida de peces amazónicos de consumo humano. Universidad Nacional de la Amazonía Peruana. Consejo Nacional de Ciencia y Tecnología. Gobierno Regional de Loreto. Instituto de Investigaciones de la Amazonía Peruana. Iquitos. Perú. 15pp.

Araujo-Lima, C.; Goulding, M. 1997. So Fruitful a Fish. Ecology, conservation and Aquaculture of the Amazon's Tambaqui. Columbia University. Press. New York, US. 191pp.
Arbeláez-Rojas, G.; Machado, D.; Indrusiak, J. 2002. Composição Corporal de Tambaqui, Colossoma macropomum, e Matrinxã, Brycon cephalus, em Sistemas de Cultivo Intensivo, em Igarapé, e Semi-Intensivo, em Viveiros. Revista Brasilera de Zootecnia, 31(3): 1059-1069.

Bourne, R. 2010. Fundamentals of Digital Imaging in Medicine. Springer. New York, US. 185pp.

Correia, G.; Freitas, C. E. 2013. Relação Pesocomprimento de Colossoma macropomum e Prochilodus nigricans a partir de dados de desembarque em Manacapuru-Am. Scientia Amazonia, 2(2): 15-19.

Duttra, S. Maity, S.; Chanda, A.; Akhand, A.; Hazra, S. 2012. Length weight relationship of four commercially important marine fishes of Northern Bay of Bengal, West Bengal. India. Journal of Applied Environment and Biological Sciences. 2(2): 52-58.

Honda, E. M. S. 1974. Contribuição ao conhecimento da biología de peixes do Amazonas. II alimentação do tambaqui (Colossoma macropomum). Acta Amazónica. 4:47-53.

Kubitza, F. 2004. Coletânea de informações aplicada ao cultivo de tambaqui, pacu e de outros peixes redondos. (http://www.panoramada aquicultura.com.br). Acceso: 10/12/2015.

Lovshin, L. 1980. Progress report on fisheries development in northeast Brazil. International Center for Aquaculture. Agricultural Experiment Station. Auburn University. Research and Development Series. No. 26. Auburn AL, US. 15pp.

Morris, A. 2005. Length-weight relationships for twenty marine fishes of Texas. Management Data Series. No 227. Sport Fish Restoration. Texas Parks \& Wildlife. National Oceanic And Atmospheric Administration. Texas, US. 11pp.

Muto, E., Soares, L.; Rossi-Wongtschowski, C. 2000. Length-weight Relationship of marine fish species of São Sebastião system, São Paulo, Southeastern Brazil. Naga, The ICLARM Quarterly. 23(4): 27-29.

Nehemia, A.; Maganira, J.; Rumisha, C. 2012. Length-weight relationship and condition factor of tilapia species grown in marine and fresh water ponds. Agriculture and Biology Journal of North America. 3(3): 117-124.

Ricker, E. 1975. Computation and interpretation of biological statistics of fish populations. Environment. Fisheries and Marine Service. Canada. Bulletin. 191:207-215. 
Sousa, R.G.; Prado, G.F.; Piñeiro, J.I.; Bezerra, E.B. 2016. Avaliação do ganho de peso do tambaqui cultivado com diferentes taxas de proteínas na Alimentação. Biota Amazonia. Open Journal System. Disponível em http://periodicos.unifap. br/index.php/biota. Acceso: mayo 2016.

Stewart, D.; Benz, G.; Scholten, G. 2009. Weightlength relationships and growth data for Blue
Catfish from four Tennessee waterbodies. Proceedings of the Southeastern Association of Fish and Wildlife Agencies. 63: 140-146. Vazzoler, E. 1982. Manual de métodos para estudos biológicos de populações de peixes. Reprodução e crescimento. CNPq. Brasilia, Brasil. 108pp.

Recibido: 21 de Enero del 2016

Aceptado para publicación: 26 de Febrero del 2016 